\title{
Validación de una escala para medir el bienestar subjetivo de los médicos (BISUMED)
}

\author{
NINA HORWITZ ${ }^{\text {la }}$, LUZ BASCUÑÁN ${ }^{1 \mathrm{~b}}$, IRENE SCHIATTINO ${ }^{2 c}$, \\ JULIA ACUÑA ${ }^{1}$, JUAN PABLO JIMÉNEZ ${ }^{1}$
}

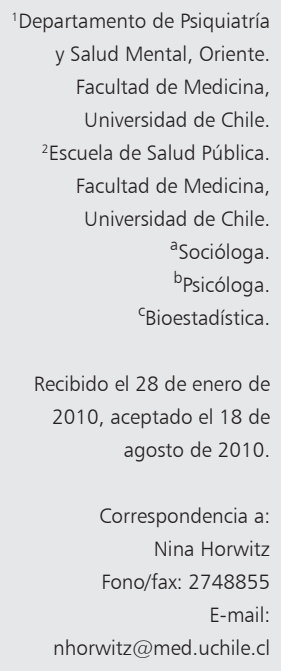

'Departamento de Psiquiatría y Salud Mental, Oriente. Facultad de Medicina, Universidad de Chile. 2Escuela de Salud Pública. Facultad de Medicina, Universidad de Chile. asocióloga. bsicóloga. cBioestadística.

Recibido el 28 de enero de 2010, aceptado el 18 de agosto de 2010.

Correspondencia a: Nina Horwitz Fono/fax: 2748855 E-mail: nhorwitz@med.uchile.cl

\section{Generation and validation of a scale to measure physicians' wellbeing}

Background: The recent and ongoing changes in the structure and social organization of medicine have deeply transformed medical practice. Aim: To study the perception of these changes by physicians, the impact of these changes in their subjective wellbeing and their strategies of adjustment. Material and Methods: A scale, consisting of 54 items grouped in nine dimensions to measure physicians' subjective wellbeing was devised. It was applied to a random sample of 580 physicians residing in Metropolitan Santiago and affiliated to the Colegio Médico de Chile (the Chilean Medical Association). Results: The internal consistency analysis in the instrument showed a global Cronbach's alpha of 90 percent. Conclusions: These results support our methodological approach based on an initial qualitative identification of relevant topics in our local context, which afterwards were included as items in the scale to measure specific components of subjective wellbeing.

(Rev Med Chile 2010; 138: 1084-1090).

Key words: Happiness; Mental health; Physicians.

\section{L}

os cambios en la estructura y organización social de la práctica médica ocurridos en ejercicio de la medicina, agregándole nuevas presiones así como oportunidades. Existe evidencia internacional sobre el impacto subjetivo que estas transformaciones han tenido en la vida profesional y personal de los médicos, es decir, en su identidad profesional y en el compromiso emocional con el que invisten su práctica médica, así como también en su vida personal. Interesante resulta destacar los planteamientos de N. Edwards y cols ${ }^{1}$ y de R. Smith ${ }^{2}$ en este sentido, quienes entienden el malestar de los médicos como respuesta a un cambio de orden mayor en el pacto psico social entre la profesión, los empleadores, los pacientes y la sociedad.

El interés por investigar el bienestar y la salud física y mental de las personas, se fue incremen- tando a partir de la década de 1980-90, en contraste con un foco anteriormente centrado en la incapacidad, la enfermedad y la patología ${ }^{3}$. Este campo da cabida a una línea de trabajo teórico y empírico asociado con los estudios relativos a la felicidad y la calidad de vida. La pregunta por la felicidad, de raigambre filosófica, se plantea no sólo a nivel individual sino también colectivo. De este modo, ha existido un importante esfuerzo empírico destinado a evaluar tanto cualitativa como cuantitativamente el bienestar, la felicidad, la satisfacción con la vida y el afecto positivo ${ }^{4-7}$.

El bienestar subjetivo se refiere a las evaluaciones positivas y negativas que las personas hacen de su vida involucrando juicios cognitivos y la experiencia afectiva ${ }^{7}$. Incluye los ámbitos de satisfacción con la vida personal, laboral y la salud, así como percepciones o juicios globales de satisfacción vital. Los trabajos en esta área concuerdan 
en la dificultad para estimar la utilidad de escalas para evaluar preocupaciones vitales y subjetivas específicas sin haber establecido previamente el contexto general en que tales sentimientos se generan. Se reconoce así la necesidad de configurar un "mapa conceptual" de las áreas asociadas a la percepción general o de aspectos específicos del bienestar/malestar subjetivo, considerándose esta tarea como un componente esencial de los estudios exploratorios. En esta línea y ante la creciente producción de instrumentos cuantitativos en esta materia, la Organización Mundial de la Salud desarrolló la metodología de exploración etnográfica gradual o paso a paso ("stepwise etnographic exploration") como un aporte a la investigación de factores psicosociales y del comportamiento en el marco de la promoción de la salud y el desarrollo humano ${ }^{8}$. Este enfoque pretende integrar métodos de exploración y análisis cualitativos y cuantitativos, respondiendo a la mencionada falencia de instrumentos para considerar las situaciones y significados del contexto social y cultural antes de definir los ítems que componen los instrumentos.

Esta es la opción metodológica escogida en el presente trabajo que se integra en una línea de investigación iniciada en 2004 y que ha tenido como objetivo conocer el efecto que tienen en el bienestar subjetivo de los médicos, la percepción de cambios en las condiciones de su práctica profesional, así como las estrategias para adaptarse a estos cambios. En primer lugar, se realizó una investigación cualitativa que confirmó los hallazgos internacionales antes mencionados y permitió conocer la configuración de los conceptos relevantes en el ámbito chileno en relación con la percepción de los médicos sobre los cambios en su profesión, el impacto de éstos en su bienestar y las estrategias de afrontamiento para adaptarse a ellos ${ }^{9-13}$. Sobre esta base y otros modelos, se elaboró un cuestionario auto administrado para medir el bienestar subjetivo de los médicos ante los cambios percibidos en su práctica profesional (BISUMED), en el medio nacional.

El objetivo de este artículo es describir el proceso de validación del cuestionario BISUMED.

\section{Material y Método}

\section{a) Elaboración del instrumento}

Primeramente, se elaboró el cuestionario de bienestar subjetivo de los médicos (BISUMED). En este proceso, se consideraron diversos modelos e instrumentos internacionales de bienestar subjetivo $9,14-18$ así como también las temáticas recogidas en la etapa cualitativa anterior. Este primer cuestionario se sometió a una validez de apariencia a través de un juicio de expertos con el fin de eliminar ambigüedades, asegurar su comprensión y pertinencia cultural. El cuestionario original incluyó 78 ítems y su estructura se presenta en la Tabla 1.

\section{b) Diseño de la muestra}

La población objetivo estuvo compuesta por médicos que residen en la Región Metropolitana e inscritos en el registro del Colegio Médico de Chile, por ser éste el universo conocido más inclusivo y accesible en el momento del estudio. Se realizó una estratificación del universo de 6.707 médicos según las siguientes tres variables que surgieron como especialmente relevantes en el estudio cualitativo realizado previamente: sexo, especialidad y año de ingreso al Colegio Médico, como proxy de la edad ${ }^{19}$. Estas variables serán utilizadas en un análisis estratificado posterior al presente estudio.

Tabla 1. Estructura original del cuestionario BISUMED

\begin{tabular}{|l|lll|}
\hline $\begin{array}{l}\text { Caracterización de la } \\
\text { persona }\end{array}$ & \multicolumn{2}{l|}{ Edad, sexo, situación familiar y laboral } \\
$\begin{array}{l}\text { Percepción de cambios y nivel } \\
\text { de satisfacción }\end{array}$ & Sección 1 & $\begin{array}{l}\text { Percepción de cambios en el ejercicio de la } \\
\text { profesión en los últimos diez años }\end{array}$ & $\begin{array}{l}16 \text { ítems } \\
\text { Escala Likert } 3 \text { niveles }\end{array}$ \\
\cline { 2 - 4 } & Sección 2 & $\begin{array}{l}\text { Actitud asumida por el médico frente a los } \\
\text { cambios percibidos }\end{array}$ & 14 ítems \\
& Sección 3 & $\begin{array}{l}\text { Satisfacción con distintos aspectos de la } \\
\text { vida (personal, familiar, social y laboral) }\end{array}$ & 48 ítem \\
& & Escala Likert 5 niveles \\
\hline
\end{tabular}


Tabla 2. Composición de la muestra

\begin{tabular}{|llcc|}
\hline Variables de estratificación & \multicolumn{2}{c|}{ Muestra } \\
& & Casos & \% \\
\hline Sexo & Hombre & 408 & 70,3 \\
& Mujer & 172 & 29,7 \\
Año de ingre- & 1900 a 1975 & 176 & 30,3 \\
so al Colegio & 1976 a 1993 & 236 & 40,7 \\
Médico & 1994 a 2006 & 168 & 29,0 \\
& & & \\
Especialidad & Pediatría & 130 & 22,4 \\
& Cirugía & 121 & 20,9 \\
& Medicina general & 87 & 15,0 \\
& Medicina interna & 112 & 19,3 \\
& Otra especialidad & 130 & 22,4 \\
\hline Total & & 580 & 100,0 \\
\hline
\end{tabular}

Se utilizó una muestra probabilística de 580 profesionales con un nivel de confianza de $95 \%$ y suponiendo una varianza máxima $\left(\mathrm{p}^{\star} \mathrm{q}=0,25\right)$.

\section{c) Recolección de datos}

Para recoger los datos se editó el cuestionario auto administrado en agenda electrónica y en papel. La tasa de respuesta fue de $43 \%$ y la de rechazo de $27 \%$.

Se utilizó un procedimiento de consentimiento informado tanto en forma verbal como escrita.

\section{d) Análisis de los datos}

Para el análisis de los datos se utilizó el software estadístico STATA 9.0. El plan de análisis, sobre la base de 78 ítems de la segunda parte del cuestionario, consideró un estudio de homogeneidad y poder discriminativo de cada uno de ellos, previo al análisis de validez de contenido del instrumento.

La evaluación de la homogeneidad utilizó el criterio que las respuestas para cada ítem no coincidieran en $75 \%$ o más de los casos. Para evaluar el poder discriminativo de cada ítem se aplicó el criterio de grupos contrastantes ${ }^{20}$, comparando para cada variable si los sujetos con puntajes sobre el percentil 75 mostraban diferencias significativas con aquellos bajo el percentil 25 del puntaje de la Escala. Se utilizó la prueba t de Student para diferencias de promedios. La asignación de puntajes a la escala resultó de la adición de puntuaciones de las respuestas para cada ítem.

De los criterios de validez de un instrumento se aplicó la de contenido ${ }^{21}$, mediante un análisis factorial a partir de los factores principales con rotación "varimax". Se evaluó la adecuación del modelo factorial mediante el índice de KaiserMeyer-Olkin (KMO). Siguiendo el criterio de Stevens $^{22}$, cada factor está conformado por los ítems con coeficientes de saturación mayor o igual a 0,40 . La confiabilidad del instrumento fue calculada usando el estadístico alfa de Cronbach. Una vez determinados los factores no se realizaron comparaciones con otros instrumentos por no contar con otras escalas o cuestionarios equivalentes, lo cual explica también por qué no se aplicó validez de criterio.

\section{Resultados}

Un total de 9 ítems fueron excluidos del instrumento y del posterior análisis de validación por no cumplir con los criterios de homogeneidad y de capacidad discriminativa.

La validez de contenido mediante el análisis factorial, consideró una solución con 9 factores (valores propios mayores o iguales a uno) y la adecuación del modelo factorial mediante el índice de Kaiser-Meyer-Olkin (KMO) fue 0,80 indicativo de buena ínter correlación según la escala de este estadístico ${ }^{23}$. Al interior de cada uno de los 9 factores se seleccionaron los ítems con un mayor coeficiente de saturación según el criterio establecido $(\geq 0,4)$. Quince ítems o afirmaciones no cumplieron con el criterio.

De esta manera, el instrumento definitivo consta de 54 ítems. Este modelo de 9 factores explicaría el $82 \%$ de la variabilidad total de la escala definitiva. En la Tabla 3 se presenta cada uno de estos factores formado por los ítems con el mayor coeficiente de saturación en el factor. Se indica el total de ítems, la varianza explicada y la consistencia interna de cada uno de los factores obtenidos (alpha de Cronbach) y en la escala propuesta. Los factores se describen en orden descendente según la varianza explicada por cada uno.

El factor 1, bienestar general, explica la mayor proporción de la varianza total $(20,6 \%)$. En él se incluyen elementos de satisfacción con la vida en general y con el logro de metas personales, con la satisfacción y la percepción de desarrollo profesional y laboral. El factor 2 reúne problemas relacionados con la salud personal, en que prima la preocupación por situaciones emocionales 
Tabla 3. Estructura factorial (con rotación varimax) del cuestionario final

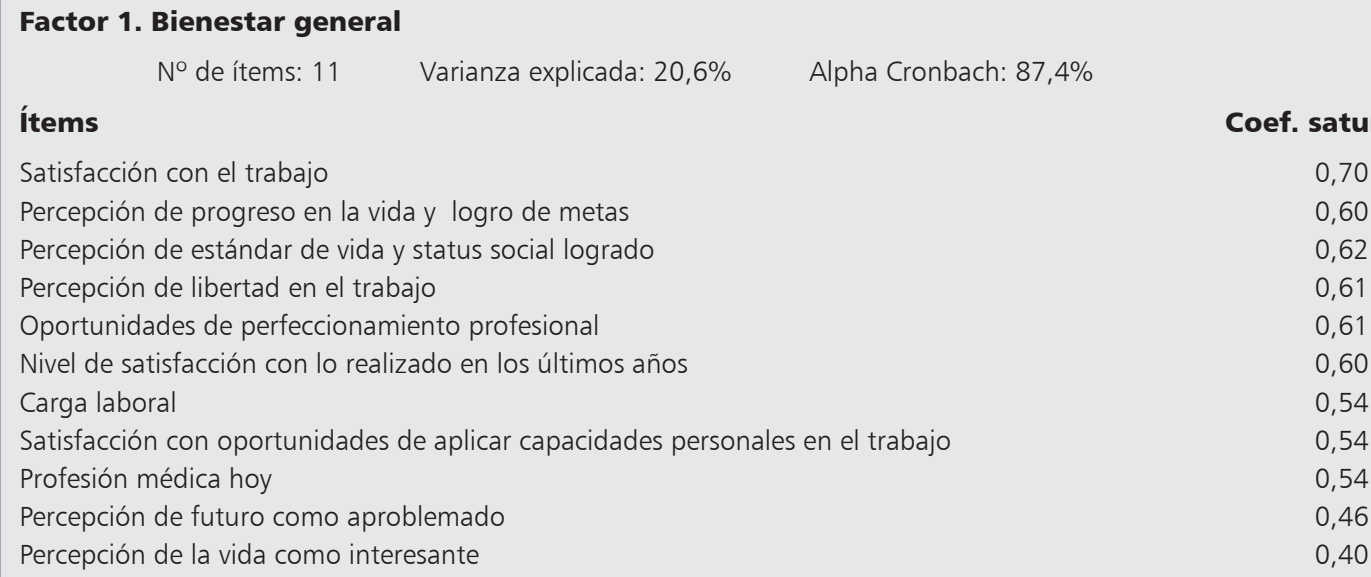

\section{Factor 2. Percepción de salud física y emocional}

№ de ítems: $10 \quad$ Varianza explicada: 13,6\% Alpha Cronbach : 83,6\%

Ítems

Coef. saturación

Sentirse tenso (a) o ansioso (a)

0,73

Sentirse irritable

Perder la paciencia ante pequeñeces

$\begin{array}{lc}\text { Frustración si las cosas no resultan como son esperadas } & 0,58\end{array}$

Sentirse triste sin razón

$\begin{array}{lr}\text { Dolores en alguna parte del cuerpo } & 0,50\end{array}$

$\begin{array}{ll}\text { Molestia ante críticas } & 0,48\end{array}$

$\begin{array}{ll}\text { Cansancio } & 0,48\end{array}$

$\begin{array}{lr}\text { Mantener la concentración } & 0,44\end{array}$

$\begin{array}{lr}\text { Problemas para dormir } & 0,44\end{array}$

Factor 3. Percepción de confianza en uno mismo y autonomía

\begin{tabular}{lcc}
\multicolumn{1}{c}{$\mathrm{N}^{\circ}$ de ítems: $4 \quad$ Varianza explicada: $9 \%$} & Alpha Cronbach: 79,4\% & Coef. saturación \\
Confianza en coraje para enfrentar crisis vitales & 0,72 \\
Confianza en capacidad para enfrentar el futuro & 0,67 \\
Capacidad de enfrentar situaciones inesperadas & 0,60 \\
Logro de metas personales & 0,45
\end{tabular}

\section{Factor 4. Percepción de cambios en el ejercicio de la profesión}

No de ítems: $9 \quad$ Varianza explicada: 7,9\% Alpha Cronbach: $68,9 \%$

Ítems

Coef. saturación

En la introducción de variables legales en la práctica profesional 0,59

En el nivel de intervención de profesionales no médicos en la atención de salud $\quad 0,50$

$\begin{array}{lr}\text { En la introducción de aspectos administrativos y de gestión } & 0,49\end{array}$

$\begin{array}{lr}\text { En el nivel de información de los pacientes } & 0,48\end{array}$

En el nivel de confianza mutua en la relación médico-paciente $\quad 0,43$

$\begin{array}{ll}\text { En el nivel de exigencia y expectativas de los pacientes } & 0,43\end{array}$

Protocolos de atención y guías clínicas basadas en evidencia (AUGE) $\quad 0,40$

En el nivel de autonomía y control del médico en el ejercicio de la profesión $\quad 0,40$

$\begin{array}{lr}\text { Defensa institucional frente a problemas médicos legales (Auge) } & 0,40\end{array}$ 
Tabla 3. Estructura factorial (con rotación varimax) del cuestionario final (continuación)

\begin{tabular}{lc}
\hline $\begin{array}{l}\text { Factor 5. Espiritualidad y trascendencia } \\
\text { No de ítems: } 5 \quad \text { Varianza explicada: 7,4\% }\end{array}$ & Alpha Cronbach: 63,5\% \\
Ítems & \\
Sentimiento de ser parte de un todo armónico & 0,51 \\
Apoyo en valores espirituales & 0,40 \\
Elección de la profesión nuevamente & 0,45 \\
Sentimiento de ser parte de un proyecto colectivo (religioso, político, deportivo) & 0,40 \\
Sentido de la vida & 0,40
\end{tabular}

Factor 6. Relaciones interpersonales

\begin{tabular}{lcc}
\multicolumn{1}{c}{$N^{\circ}$ de ítems: 4} & Varianza explicada: 7,2\% & Alpha Cronbach: $68,4 \%$ \\
Ítems & Coef. saturación \\
Sentimiento de problemas en la relación con su pareja & 0,68 \\
Sentimiento de problemas en la relación con hijos & 0,66 \\
Falta de un buen amigo & 0,53 \\
Necesidad de más amigos & 0,50
\end{tabular}

\begin{tabular}{|c|c|c|c|}
\hline No de ítems: 4 & Varianza explicada: $5.6 \%$ & Alpha Cronbach: $68.7 \%$ & \\
\hline Ítems & & & Coef. saturación \\
\hline Reconocimiento por pares & & & 0,64 \\
\hline Relaciones con colegas & & & 0,57 \\
\hline Reconocimiento por jefes & & & 0,53 \\
\hline Reconocimiento por pacientes & & & $0,28 *$ \\
\hline
\end{tabular}

Factor 8: afrontamiento activo y reflexivo

No de ítems: $4 \quad$ Varianza explicada: 5,3\% Alpha Cronbach: 37,5\%

ítems

Coef. saturación

$\begin{array}{ll}\text { Búsqueda de ayuda ante problemas en el trabajo } & 0,42\end{array}$

$\begin{array}{ll}\text { Reflexión ante problemas en el trabajo } & 0,41\end{array}$

$\begin{array}{ll}\text { Búsqueda de nuevas herramientas para entender cambios } & 0,41\end{array}$

$\begin{array}{ll}\text { Afrontamiento de cambios ampliando actividades profesionales } & 0,40\end{array}$

Factor 9. Afrontamiento revalorizando la medicina y la vocación de servicio

No de ítems: $3 \quad$ Varianza explicada: 5,3\% Alpha Cronbach: 52,5\%

$\begin{array}{lr}\text { Rescate de lo esencial de la medicina para enfrentar cambios } & 0,47\end{array}$

$\begin{array}{lr}\text { Apoyo en vocación de servicio para enfrentar cambios } & 0,47\end{array}$

$\begin{array}{ll}\text { Estudio y perfeccionamiento para enfrentar cambios } & 0,40\end{array}$

Alpha Cronbach global de la Escala: $90.2 \%$

Varianza explicada total: $82 \%$

*Este ítem se conservó pese a que su coeficiente de saturación es inferior al punto de corte, por consideraciones conceptuales.

de irritabilidad, tensiones y frustraciones. El factor 3 se refiere a percepción de capacidad de afrontamiento de crisis, así como auto confianza, enfrentamiento del futuro y realización de las propias metas. El factor 4 integra diferentes aspectos en los cuales pueden percibirse cambios en el ejercicio de la práctica médica. El factor 5 apunta a la vivencia de que la vida está ligada a proyectos $\mathrm{y}$ valores que superan la propia individualidad. Los factores 6 y 7 exploran las relaciones interpersonales, correspondiendo al primero aquellos vínculos afectivos cercanos e íntimos y al segundo 
Escala para medir el bienestar subjetivo de los médicos (BISUMED) - N. Horwitz et al

la dimensión laboral, especialmente en relación a la valoración y reconocimiento profesional. Los dos últimos factores se refieren a las características del afrontamiento desplegado ante los cambios en la profesión; en el factor 8 se busca afrontar los cambios saliendo de si mismo en tanto que en el factor 9 el afrontamiento se centra en la profesión médica propiamente tal, ya sea como vocación, o perfeccionamiento.

\section{Discusión}

Los cambios y reformas que están ocurriendo en la organización de la medicina y en la entrega de los servicios de salud, no sólo tienen implicaciones políticas, económicas y organizacionales. Es necesario considerar además, el impacto psicosocial sobre los profesionales de salud. Existe un interés creciente por utilizar indicadores de bienestar subjetivo en la discusión y la evaluación de políticas públicas, ya que brindan información que trasciende el alcance habitual de los indicadores económicos y sociales ${ }^{24}$. Nuestra línea de investigación, focalizada inicialmente en los médicos, ha desarrollado el cuestionario BISUMED para evaluar su bienestar subjetivo ante los cambios percibidos en el ejercicio de su profesión, entre los cuáles las reformas de salud ocupan un lugar destacado.

Los conceptos de bienestar subjetivo, calidad de vida, satisfacción y felicidad, pese a sus diferencias, están relacionados entre si y presentan importantes espacios de convergencia. Barrien$\operatorname{tos}^{25}$ menciona, entre otros, tres que nos parecen relevantes. El primero, es la dimensión subjetiva, el segundo, la distinción que en todos se encuentra entre dos aspectos estructurales: los emocionales y afectivos por una parte y los cognitivos y valorativos por otra. El último aspecto se refiere a la connotación de inclusión o el uso como término general (por ejemplo, "calidad de vida global"; "bienestar general"). Este último rasgo ha sido también fuente de problemas, dada la dificultad que existe para acordar criterios comunes para definir tales conceptos en forma genérica. Debido a esto, la tendencia creciente se centra en lograr criterios cada vez más precisos para ámbitos específicos, como es el caso del presente trabajo, que se aboca a describir el bienestar subjetivo de los médicos ante los cambios en su profesión.
En este trabajo se ha presentado el estudio de validación de un cuestionario de bienestar subjetivo de los médicos ante los cambios en su práctica profesional (BISUMED), a través del análisis de apariencia y contenido (factorial), en una muestra de 580 médicos de la Región Metropolitana de Santiago de Chile. Los resultados específicos que se obtengan del análisis de los datos de la muestra de 508 médicos, como por ejemplo, diferencias en los puntajes en los factores e ítems de acuerdo a sexo, edad y especialidad médica, serán objeto de publicaciones ulteriores.

Con el estudio de validación, se obtiene un cuestionario de 54 ítems. El análisis de la consistencia interna arrojó resultados favorables, alpha de Cronbach global de 90\%. El cuestionario aborda dimensiones específicas que aluden a la percepción de los cambios en la práctica profesional, del bienestar personal y de las estrategias para enfrentar los cambios, definidos como los tres principales ejes de estudio desde el inicio de esta línea de investigación. El resultado favorable respecto de la consistencia interna del instrumento constituye además un respaldo al enfoque metodológico elegido, ya que la elaboración del cuestionario BISUMED se inició con un estudio cualitativo exploratorio que permitió identificar los temas relevantes, los que fueron sometidos a juicio de expertos logrando una validez de apariencia ajustada a los objetivos planteados. Este proceso, que contempla una indagación conceptual previa a la formulación de los ítems y su posterior verificación estadística, ha sido descrito como una exploración etnográfica gradual. La composición definitiva de la escala BISUMED, con 54 ítems agrupados en 9 factores que explican el 82 por ciento de la variación total de los datos, resultó de un interesante intercambio entre consideraciones estadísticas y conceptuales. La complejidad del concepto de bienestar subjetivo de los médicos, en este caso, se refleja en la estructura factorial del instrumento, cuyos resultados deben interpretarse a la luz de diversas dimensiones con alto grado de autonomía específica, particularmente en el caso de los Factores 1, 2 y 3 referidos a la percepción de bienestar general, de salud física y emocional y de auto confianza respectivamente, que cuentan con un puntaje Alpha igual o superior al 80\%.

El cuestionario reúne elementos vinculados a estrés, satisfacción, valores propios del trabajo y relaciones interpersonales significativas, los cuales 
han sido motivo de estudios a lo largo de los últimos treinta años. Sin embargo, por lo general, tales estudios se han realizado a través de instrumentos diferentes. BISUMED permitiría la evaluación de estas dimensiones en un instrumento único, constituyéndose de este modo, en un aporte para el desarrollo de estudios en esta línea.

La consideración de la situación de los profesionales de salud, desde su auto percepción, contribuye a configurar una visión más compleja de los cambios en la organización social de la salud incorporando a todos los actores relevantes en la integración de las diversas aspiraciones e intereses en la política pública. El bienestar de los médicos y de los profesionales de la salud en general, no sólo los afecta a ellos, sino que es un factor vital en la calidad de la atención de los pacientes. El instrumento, cuya validación se ha presentado, es una herramienta útil y versátil ya que puede ser utilizada en su versión total o en forma segregada, centrándose en los aspectos (factores) que interesen, de acuerdo a las circunstancias.

\section{Referencias}

1. Edwards N, Kornacki MJ, Silversin J. Unhappy doctors: what are the causes and what can be done? BMJ 2002; 324: 835-8.

2. Smith R. Why are doctors so unhappy? There are probably many causes, some of them deep. BMJ 2001; 322: 1073-4.

3. Seligman M. La auténtica felicidad. Vergara, Grupo Zeta, Barcelona, España, 2003.

4. Diener E. Subjective wellbeing. Psychological Bulletin 1984; 95: 542-92.

5. Diener E, Larsen E. The experience of emotional wellbeing. En: Lewis M, Havilland RJ. (eds.) Handbook of emotions. New York; Guilford Press, 1993; p. 405-15.

6. Páez D. Afectividad, estrés y apoyo social: revisión conceptual y meta-análisis de investigaciones psicosociales realizadas en Chile. Citado en Barrientos J. Calidad de vida. Bienestar subjetivo: una mirada psicosocial. Santiago, Chile Universidad Diego Portales, 2005.

7. Schwartzman L. Calidad de vida relacionada con la salud. Aspectos conceptuales. Cienc. enferm. v.9 n.2 Concepción dic. 2003. Versión On-line ISSN 0717-9553.

8. Eid M, Diener E. Global judgments of subjective wellbeing: Situational variability and long-term stability. Social Indicators Research 2004; 65: 245-77.

9. OMS, South-East Asia. Assessment of subjective wellbeing. The subjective well-being inventory. Regional
Paper, SEARO; 1992, no 24.

10. Jiménez de la Jara JP. Bienestar y satisfacción de los médicos con el ejercicio de su profesión en un sistema de salud en proceso de reforma. Rev Med Chile 2004; 132: 635-6.

11. Jiménez de la Jara J. Cambios en el entorno del trabajo médico. Rev Med Chile 2004; 132: 637-42.

12. Horwitz N. El cambio de la práctica médica: Desafíos psicosociales para la profesión. Rev Med Chile 2004; 132: 768-72.

13. Bascuñán ML. Cambios en la relación médico-paciente y nivel de satisfacción de los médicos. Rev Med Chile 2005; 133: 11-6.

14. Jiménez de la Jara JP. Estrategias de los médicos para hacer frente a la crisis de la profesión. Rev Med Chile 2005; 133: 707-12.

15. OMS WHOQOL. Measuring Quality of life: The World Health Organization Quality of life instruments (The WHOQOL-100 and the WHOQOL-BRIEF). Division of Mental Health and Prevention of Substance Abuse. 1997.

16. Diener E, Emmons RA, Larsen RJ, Griffin S. The Satisfaction with Life Scale. Journal of Personality Assessment 1985; 49: 71-5.

17. Karasek RA, Theorell T. Healthy work: productivity and the reconstruction of working life. New York, Basic Books, 1990.

18. Kristensen TS, Borritz M, Villadsen E, Christensen KB. The Copenhagen Burnout Inventory: a new tool for the assessment of burnout. Work Stress 2005; 19: 192-2007.

19. Maslach C, Jackson SE, Leiter MP. Maslach Burnout Inventory manual ( $3^{\text {a }}$ ed.) Palo Alto, California: Consulting Psychologists Press, 19919. Departamento De Economía, Universidad De Chile. Centro De Micro Datos. Informe final Encuesta Bienestar Subjetivo de los Médicos, Santiago, Chile, Septiembre 2007.

20. Anastasi A, Urbina S. Tests psicológicos. México: Prentice Hall, 1990.

21. Sánchez R, Echeverry J. Validación de escalas de medición de salud. Rev Salud Pública, Bogotá 2004; 6: 302-18.

22. Martínez Arias R. Psicometría: teoría de los tests psicológicos y educativos. Madrid: Editorial Síntesis, 1996.

23. Reymont R, Jöreskog KG. Applied factor analysis in the natural sciences. Cambridge University Press, 1996.

24. Diener E. Guidelines for national indicators of subjective well-being and ill- being. Applied Research in Quality of Life 2006; 1: 151-7.

25. Barrientos J. Calidad de vida. Bienestar subjetivo: una mirada psicosocial. Santiago, Chile: Universidad Diego Portales, Vicerrectoría Académica/ Dirección de Extensión y Publicaciones; 2005. 